\title{
Current status of the design of the ITER bolometer diagnostic
}

\author{
H. Meister ${ }^{\mathrm{a}}$, C. Gliss ${ }^{\mathrm{a}}$, D. Hermann ${ }^{\mathrm{a}}$, M. Kannamüller ${ }^{\mathrm{a}}$, J. Kolla ${ }^{\mathrm{a}}$, G. Nadasi ${ }^{\mathrm{b}}$, A. Pataki ${ }^{\mathrm{a}}$, F. Penzel ${ }^{\mathrm{a}}$, T. Sehmer ${ }^{\mathrm{a}}$, L.

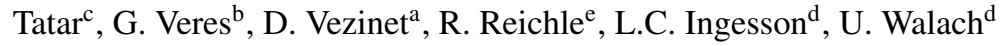 \\ ${ }^{a}$ Max-Planck-Institut für Plasmaphysik, Boltzmannstr. 2 D-85748 Garching, Germany \\ ${ }^{b}$ Wigner Research Centre for Physics, Hungarian Academy of Sciences, Konkoly-Thege Miklós 29-33, H-1121 Budapest, Hungary \\ ${ }^{c}$ Centre for Energy Research, Hungarian Academy of Sciences, Konkoly-Thege Miklós 29-33, H-1121 Budapest, Hungary \\ ${ }^{d}$ Fusion for Energy, c/ Josep Pla, $n^{\circ}$ 2, 08019 Barcelona Barcelona, Spain \\ e ITER Organisation, Route de Vinon-sur-Verdon, CS 90 046, 13067 St. Paul Lez Durance Cedex, France
}

\begin{abstract}
The ITER bolometer diagnostic shall provide the measurement of the total radiation emitted from the plasma, a part of the overall energy balance. 550 lines-of-sight (LOS) will be installed in ITER observing the whole plasma from many different angles to enable reliable measurements and tomographic reconstructions of the spatially resolved radiation profile. The LOS are bundled in 71 individual cameras, which will be located behind blanket modules on the vacuum vessel wall, in five divertor cassettes, in two upper port plugs and in one equatorial port plug. For all major design issues solutions have been elaborated and will be presented.

The design of collimators has been developed and tested on prototypes to provide an exact definition of the required viewing cones of about $1^{\circ}$ while reliably reducing reflections, stray light and microwave stray radiation. A 3D-shaped ceramic printed circuit board is proposed to hold the sensor, orient it as desired, and provide good thermal contact as well as the bridge for electrically connecting external signal cables to the meanders on the sensor. The design of the camera housing for vacuum vessel and divertor cameras has been optimised for improved management of the thermal heat flow, supported by tests defining material properties and verifying analysis. Additionally, methods have been developed to derive the main design parameters of cameras and decide if pinhole or collimator type is more advantageous. Recently, the system-level design phase started and uses the achieved results to define interfaces and designs for the specific locations in ITER, with a focus on the vacuum-vessel cameras to finalise the interface with blanket modules.
\end{abstract}

Keywords: ITER, bolometer, design integration, plasma diagnostics

\section{Introduction}

The total radiated power as well as the radiation emission profile on ITER will be determined by the bolometer diagnostic. A bolometer measures the plasma radiation over a wide spectral range (from soft- $X$ to the infrared) by monitoring the temperature rise induced by deposition of photon energy in the absorber layer of the bolometer. The reference detector type chosen for ITER is the metal resistor bolometer $[1,2]$. In order to determine the spatially resolved radiation profile, tomographic reconstruction routines are applied on the measurement results from many lines-of-sight (LOS).

This paper describes the current status of the design of the ITER bolometer diagnostic system. Section 2 gives a brief overview of the main diagnostic components and underlying principles to derive design param- eters. Section 3 focuses on $R \& D$ results for two generic components, the collimator and the sensor support. The design of camera housings has to be adapted according to their locations in the ITER vessel. Main results for the design integration in ITER are given in Section 4 before concluding with an outlook on next steps.

\section{Overview of the ITER bolometer diagnostic}

A typical bolometer collimator camera is shown in Figure 4, which highlights the main components: the sensor to acquire the signal proportional to the incident plasma radiation, the collimator to accurately define the LOS, the 3D-shaped sensor support to hold the sensor in the correct position and orientation as well as provide the thermal path to the supporting structure and to bridge the electrical connection between external signal 
cables and the sensor. Finally, the housing encloses everything and takes up the external loads and the mounting platform attaches the camera to the supporting structure.

Inside the ITER vessel diagnostic components will be subjected to a variety of loads. Depending on their location they will have to withstand varying thermal loads due to neutron and gamma heating (volumetric heating up to $0.5 \mathrm{~W} / \mathrm{cm}^{3}$ ) as well as from plasma radiation and $\mathrm{ECH}$ stray radiation (surface loads up to $35 \mathrm{~kW} / \mathrm{m}^{2}$ ), mechanical loads due to $E \times B$ forces during plasma disruptions and operate in vacuum at elevated temperatures.

The ITER bolometer diagnostic will consist of 550 individual LOS. They are defined by 71 cameras which are distributed toroidally and poloidally over the ITER vacuum vessel. Figure 1 shows the LOS mapped into one poloidal plane on the left and a schematic of the toroidal distribution on the right. A high density of LOS
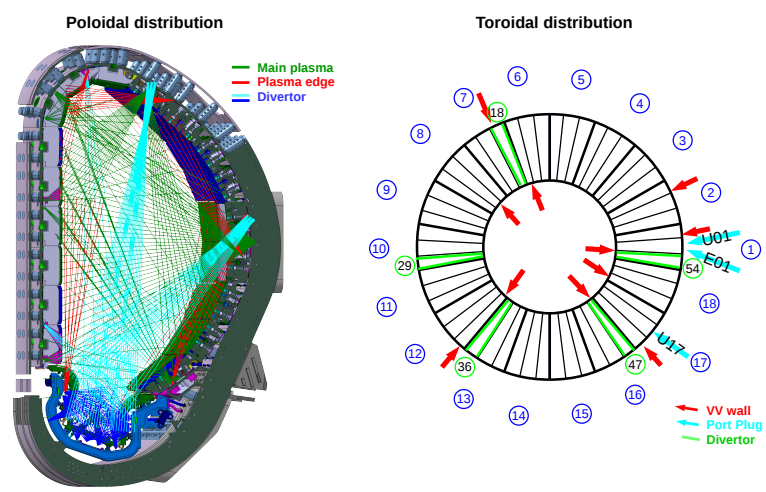

Figure 1: ITER bolometer LOS mapped into one poloidal plane (left) and a schematic of the toroidal distribution (right).

is gathered within sectors 1 and $16-18$ providing the main data for tomographic reconstructions. Additionally, LOS in the sectors 7 and 12 provide information on any potential asymmetry in the toroidal distribution of the plasma radiation, which will be used in particular for the optimisation of the disruption mitigation system.

For an accurate tomographic reconstruction the fields of view of each LOS need to be known. They have to be defined such that the resulting viewing cones cover all plasma regions from various angles [3]. As diagnostic components in ITER have to be placed behind blanket modules $(\mathrm{BM})$ providing neutron shielding the resulting LOS will have to define viewing angles in the order of a very few degrees. The LOS can be defined either by collimators or by pinhole cameras. Methods to derive the main design parameters of these camera types are described in [4] in detail. The camera type can be cho- sen according to the available construction space and the resulting étendue.

The sensor development efforts, which are ongoing in parallel and aim towards a sensor able to withstand temperatures up to $400^{\circ} \mathrm{C}$, suggest that the heat capacity will increase due to the increase of absorber thickness by a factor of two. The cooling time constant will depend on the success of optimisation efforts but can be expected to range between $200 \mathrm{~ms}$ and $750 \mathrm{~ms}$. The sensitivity and spectral efficiency are to be determined once first prototypes with the required absorber thickness of $20 \mu \mathrm{m}$ are available. Based on the experience with currently operated bolometer systems the AC excitation of the Wheatstone bridge for bolometer data acquisition and the resulting measured voltages in the range of a few $\mathrm{mV}$ suggest that the data acquisition hardware should be placed as close as possible to the sensor while still avoiding the harsh nuclear environment close to the ITER vessel. A compromise was found by placing the hardware within shielded cubicles in the gallery or within the shielded corners of the tokamak building, as close as possible to the electrical feedthroughs at the ports. Thus, total cable lengths could be limited to about $80 \mathrm{~m}$. This is still close to a factor of two longer than used on current devices and is thus proposed to be tested on prototypes.

\section{Design development of generic components}

\subsection{Collimators}

The majority of bolometer cameras in ITER will be of the collimator type in order to define small viewing cones within a restricted space envelope. Additionally, stray light and reflections have to be minimised and ECH stray radiation shall be prevented from reaching the sensor.

The definition of the main parameters of the collimator (length, no. of sub-collimators, dimensions of pinholes) are calculated according to [4]. However, the thickness of apertures inside the collimator, their number and exact placement determines the efficiency of reducing stray light and reflections. In particular, collimators divided into sub-collimators to reduce the viewing cone in toroidal direction will suffer from cross-talk of light between adjacent sub-collimators if the design is not well chosen. A schematic of such a collimator is shown in Figure 2 on the left. First measurements of the transmission function of collimator prototypes have been made using a robot and a laser to illuminate the entrance aperture of the collimator from various angles 


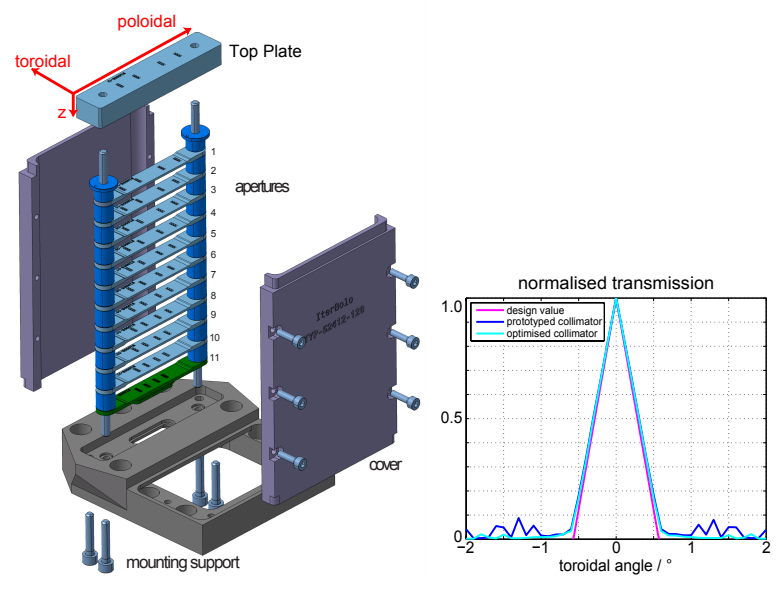

Figure 2: Left: Schematics of a collimator indicating main components. Right: Comparison of MCRT results of normalised transmission function for prototyped collimator as given on left and optimised design w.r.t. expected values.

[5]. Observed discrepancies to the expected transmission function according to design data have been analysed by Monte Carlo Raytracing (MCRT) techniques [6]. MCRT simulations were benchmarked to the experimental data to determine the material properties for reflections. The simulation results demonstrated that some space between apertures and around the holes for the LOS in the apertures is necessary to disperse stray light from reflections on the surface of the apertures. Additional deviations of the expected transmission function are caused by cross-talk of light from one sub-collimator to an adjacent one and by reflections on the inner edges of apertures. The former effect can be reduced by increasing the number of apertures and their thickness as this provides more effective surface to prevent cross-talk. The latter effect however, increases with the effective surface of apertures. Thus, a compromise has to be found. Running several simulations while varying the number and thickness of apertures yielded a database of transmission functions in dependence of aperture number and thickness. The amount of reflected light and stray light is minimised for one given set of parameters and determines the optimal configuration of the collimator. This method strongly depends on the number of sub-collimators and has to be performed for each collimator type individually. In case of a collimator with $120 \mathrm{~mm}$ length and 3 sub-collimators the optimisation results in 16 equidistantly spaced $0.5 \mathrm{~mm}$ thick apertures. The corresponding transmission functions are given in Figure 2 on the right. Resulting étendues for ITER are still subject to optimisation. Based on in- termediate estimations they vary from $4 \cdot 10^{-9} \mathrm{~m}^{2}$ up to $8 \cdot 10^{-8} \mathrm{~m}^{2}$ if the simplified calculation for the étendue is taken as $E=\frac{A_{s} \cdot A_{p}}{L_{C}^{2}}$ with the areas of sensor $\left(A_{s}\right)$ and pinhole $\left(A_{p}\right)$ and the length of the collimator $L_{C}$.

Additionally, methods have been investigated to reduce the impact of ECH stray radiation. As previous investigations [7] demonstrated, two methods should be combined: a top plate on the collimator including a microwave reflecting grid effectively suppresses $\mathrm{ECH}$ stray radiation and can be optimised for the $\mathrm{ECH}$ wavelength used in ITER. mm-wave radiation from the plasma with a broader frequency spectrum occurs at high plasma temperatures. It can be suppressed by applying a microwave coating on the inside of the collimator. As explained in detail in [7], attenuation factors up to $70 \mathrm{~dB}$ can be achieved.

\subsection{Sensor support, orientation and electrical connec- tion}

With the high operating temperatures of ITER the requirements on the support and electrical connection of the sensor are more demanding than for currently active devices. The support structures should offer good thermal coupling to the environment so that temperatures are kept as low as possible. Electrical connections have to withstand the operating temperatures and need to operate reliably for the life time of ITER. Finally, every sensor has its unique orientation with respect to the surface on which it is mounted.

A 3D-shaped printed circuit board (3D-PCB) is proposed as design solution to meet all above named requirements within one component. The supporting structure is made of AlN, a ceramic with high thermal conductivity $(180 \mathrm{~W} /(\mathrm{m} \cdot \mathrm{K}))$ and good insulating properties. There exist several techniques to deposit electrical tracks on a 3D shaped substrate, e.g. micro dispensing, laser activation or laser etching. For all of these techniques prototyping is ongoing to test on simplified component geometries the resulting properties. For laser etching a 3D-PCB has already been produced. Figure 3 shows a schematics of the prototype produced and indicating a sensor in its holder on the left. This geometry orients the sensor parallel to the supporting surface in order to simplify the prototype production and related costs. Including an inclination angle would be unproblematic. The same figure shows on the upper right a picture of a signal cable brazed to the contact pads and on the lower right a detail of the electrical tracks running on the back side, crossing rectangular corners and connected partially by vias to the front side. 


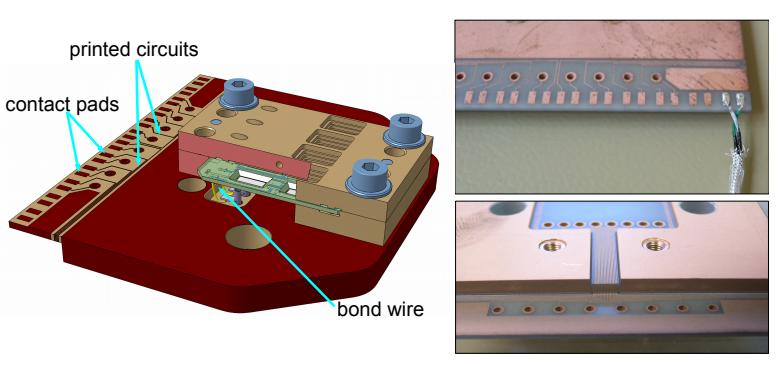

Figure 3: Schematic of a flat 3D-PCB with a sensor in its holder (left) and pictures of a prototype produced by laser etching showing a signal cable brazed to contact pads (upper right) and a detail of electrical tracks on the back side running over corners and partially connecting to front side by vias (lower right).

To reliably connect the sensor bonding has already been applied successfully for bolometers at JET [8]. Similarly, it is now proposed to use bonding for the connection from the 3D-PCB to the sensor. However, the most suitable method still needs to be developed as the meanders and contact pads on the sensor are made of $\mathrm{Pt}$ for its better resistivity against neutron irradiation than $\mathrm{Au}$, which was used previously [9]. The electrical connection from 3D-PCB to external signal cables can be brazed as shown in Figure 3 or laser welded. For all these connections attention has to be paid to the choice of materials and the operating temperature: they have to be compatible to the ITER vacuum and irradiation environment while matching Seebeck coefficients to avoid the generation of thermoelectric voltages.

The ongoing prototyping campaign will demonstrate the properties of the various manufacturing techniques for 3D-PCBs. Of particular interest will be both, the restrictions for the design of such components as well as the behaviour under thermal and neutron loads.

\section{Design integration in ITER}

The locations on which bolometer cameras will be placed in ITER can be separated into 3 generic areas, each with its own typical boundary conditions and requirements. The maturity of the respective designs is at different levels, corresponding to the time line when those components will be needed for installation.

\subsection{Cameras on vacuum-vessel}

Figure 4 shows the current design of a typical VV camera. The generic method accepted to attach components onto the VV wall are so-called bosses, studs of stainless steel welded onto the VV wall with a hole in the centre featuring a thread for bolting and used for examining the quality of the full penetration weld. As

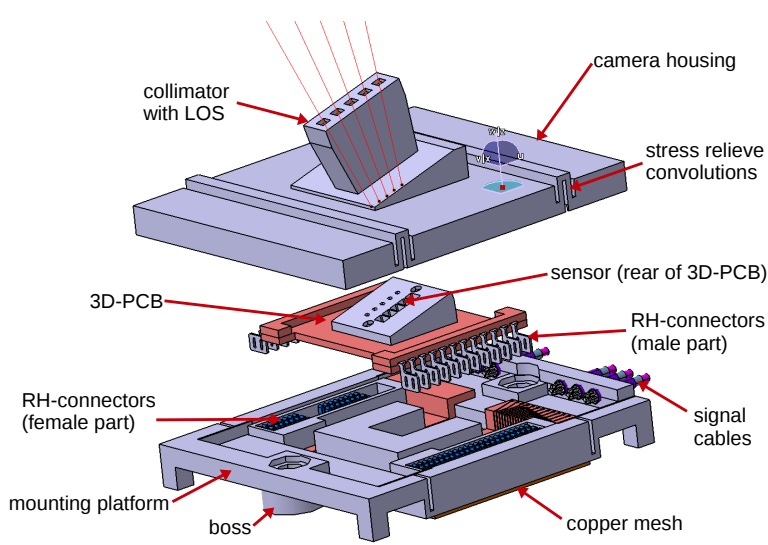

Figure 4: Typical VV bolometer camera with its main components indicated.

BMs will be installed only after first plasma and may be aligned in their position by $\pm 12.5 \mathrm{~mm}$ in both, toroidal and poloidal direction led to separating the camera in two parts: the mounting platform fixes the signal cables and is installed before first plasma. The main camera will be installed after first plasma and after customisation according to the finally defined positions of the BMs. Typical feature for RH are not yet included in the design, but space requirements are accounted for.

A major concern is the handling of loads. Stresses imposed by the thermal expansion of the VV during operating conditions are taken up by the convolutions in both mounting platform and camera housing. Using the same material for these two components assures that no additional stresses arise due to thermal gradients. The material choice is currently being investigated. The decision between the candidates TZM, a molybdenum alloy, and $\mathrm{CuCrZr}$ will be made according to the analyses results on thermal and structural behaviour. In particular, a distortion of the viewing cone and thus a change of LOS direction and reduction of the field of view has to be avoided. As no active cooling will be available for the VV cameras, a good thermal connection to the VV wall acting as heat sink is essential and realised by a copper mesh beneath the main camera body. Furthermore, the design aims at providing a direct heat flow path from sensor to VV wall and from collimator to VV wall, but separating those two as much as possible. Together with using materials with high thermal conductivity (TZM or CuCrZr for housing and platform, AIN for ceramics), current analyses estimate the temperature at the sensor to be in the order of $220^{\circ} \mathrm{C}$, which may lead to a reduced $\mathrm{S} / \mathrm{N}$-ratio due to higher operation temperatures compared to current systems, but is still well below the limit of $350^{\circ} \mathrm{C}$ sensors will have to withstand 
during baking of the divertor. Thermal material properties essential for the analyses have already been verified on prototype tests in [10].

\subsection{Divertor cameras}

Bolometer cameras in the divertor cassettes have to be placed so that they can observe the plasma through the gaps between adjacent cassettes. Thus, camera components will have to occupy the space between cassettes if the reduced light yield due to an oblique view is not wanted. This in turn subjects them to requirements imposed by the remote-handling installation process of the divertor cassettes resulting in strongly reduced space envelopes available for the cameras. Furthermore, it implies that the heat sink for the camera is the zone for attaching it to the cassette body, which is located to one side (see Figure 5).

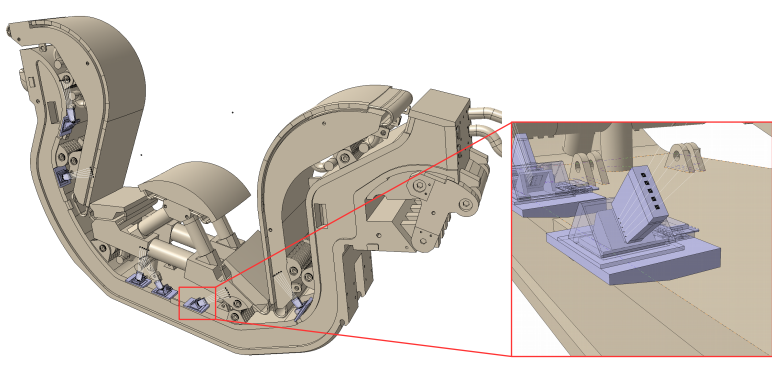

Figure 5: Typical divertor bolometer cameras attached to the side of a divertor cassette (left) and zoom onto one camera showing the parametric model and its attachment to the cassette body on one side (right)

Thermal loads result from nuclear heating and from plasma radiation. While the former is an evenly distributed volumetric load, the latter is a concentrated surface load onto the areas within the gaps. To avoid strong asymmetries in the resulting temperature pattern, in particular for the bolometer sensor, a dedicated parametric model of the camera design has been implemented and analysed [11]. For various locations it could be demonstrated that design parameters can be optimised so that the temperature gradient between measurement and reference absorber is reduced to below $1{ }^{\circ} \mathrm{C}$ and thus minimising the impact of temperature gradients on the measurement error.

Furthermore, vignetting of the viewing cone for the LOS has to be avoided. In order to avoid obscuring the viewing cone in toroidal direction, an accuracy of the camera installation of $0.1^{\circ}$ is necessary. This can be achieved e.g. by separating the camera in two parts, platform and housing as for the VV cameras. Measurements of the as installed platforms and corresponding customisation of the housing can assure this accuracy. Thermal expansions of the camera and the cassettes during operation and their impact on the gap size through which the LOS have to pass need to be taken into account during the design of the collimators in the form of tolerances for the final direction and size of the viewing cones. Their exact value still needs to be determined but may lead to reduced width and thus a reduction in the light yield. Finally, movements of different LOS w.r.t. each other might occur during operation due to thermal effects and have to be assessed as they impact the tomographic reconstruction uncertainty of the system.

\subsection{Port plug cameras}

For port plug cameras scoping studies were made to determine the type and the main design parameters of the cameras. Using the LOS distribution as proposed during the conceptual design review, the required space envelopes have been defined based on [4] and in cooperation with the port integrator. In the equatorial port plug EPP01 there are three cameras: one pinhole camera in the diagnostic shield module (DSM) no. 2 observes the main plasma with 35 LOS. In DSM 3 a pinhole camera with 15 LOS views the upper plasma and a collimator camera with 40 LOS observes with high spatial resolution the LFS edge plasma and the divertor region. Both upper port plugs equipped with bolometer cameras (UPP01 and UPP17) have identical space envelopes and camera types, but differ slightly in the orientation of the LOS. In each of them there is a pinhole camera viewing the upper X-point region with 10 LOS, a pinhole camera viewing the main plasma on the HFS with 30 LOS and the main plasma on the LFS with 10 LOS. Finally, a collimator camera with 30 LOS views the divertor region with high spatial resolution.

\section{Conclusions}

A significant effort has been invested in the conceptual design of the ITER bolometer diagnostic resulting in detailed concepts for camera types in general as well as for individual components. In particular the collimator design has been investigated in detail and verified on prototypes. Furthermore, a 3D-PCB has been proposed to support, orient and contact the sensor and its manufacturability has been verified. Future design work concentrates now on the finalisation of interface definitions with surrounding components and the detailed design. Apart from the development of the sensor, which has not been discussed here at all, a significant amount of analysis work will be necessary to validate the concepts. This 
includes in particular the nuclear analysis and MCNP simulations to derive specific loads for the bolometer cameras as well as to provide information for life-time assessments and the definition of the interfaces with radwaste management. These loads as well as those from electro-magnetic forces during disruptions need to be considered for the full structural analyses which have to iterate with the detailed design process. Finally, an increasing level of detail needs to be implemented in the design of all cameras until their integration in ITER is finalised.

\section{Acknowledgments}

This work was partly supported by funds from the German Ministry for Education and Research under the Grant No. 03FUS0006 and by Fusion for Energy under the Grant F4E-FPA-384-SG03. The views expressed in this publication are the sole responsibility of the authors and do not necessarily reflect the views of the Fusion for Energy and the ITER Organization. Neither Fusion for Energy nor any person acting on behalf of Fusion for Energy is responsible for the use, which might be made of the information in this publication.

\section{References}

[1] K. F. Mast, J. C. Vallet, C. Andelfinger, P. Betzler, H. Kraus, and G. Schramm. A low noise highly integrated bolometer array for absolute measurement of VUV and soft-X radiation. Rev. Sci. Instrum., 62(3):744-750, 1991.

[2] H. Meister, L. Giannone, L.D. Horton, G. Raupp, W. Zeidner, G. Grunda, S. Kalvin, U. Fischer, R. Reichle, A. Serikov, and S. Stickel. The ITER bolometer diagnostic - status and plans. Rev. Sci. Instrum., 79:10F511-1 - 10F511-5, 2008.

[3] L. C. Ingesson and D. J. Wilson. Optimization of apertures and collimators for multi-channel plasma diagnostics. Rev. Sci. Instrum., 73(8):2890-2899, 2002.

[4] H. Meister and S. Kalvin. Optimisation of design parameters for collimators and pin-holes of bolometer cameras. Fusion Eng. Des., 89(12):3039-3045, 2014.

[5] F. Penzel, H. Meister, L. Giannone, M. Kannamüller, J. Koll, T. Trautmann, and A.W. Koch. Assessment of line of sight characteristics of ITER bolometer prototype collimators. Fusion Eng. Des., 88(6-8):1267-1270, 2013. Proceedings of the 27th Symposium on Fusion Technology (SOFT-27); Liège, Belgium, September 24-28, 2012.

[6] N. Homner. Kollimatoroptimierung für die ITERBolometerdiagnostik mittels Monte-Carlo Raytracing. Master's thesis, Ludwig-Maximilians Universität München, 2014.

[7] H. Meister, W. Kasparek, D. Zhang, M. Hirsch, J. Koll, and A. Zeitler. Millimetre wave attenuation of prototype diagnostic components for the ITER bolometers. Fusion Eng. Des., 9697:861 - 864, 2015. Proceedings of the 28th Symposium On Fusion Technology (SOFT-28).

[8] R. Reichle, J. C. Fuchs, R. M. Giannella, N. A. C. Gottardi, H. J. Jäckel, K. F. Mast, P. R. Thomas, and P. van Belle. Bolometer for ITER, Proceedings of the International Workshop on Diagnostics for ITER held in Varenna, Italy, August 28 - September 1, 1995, Diagnostics for Experimental Thermonuclear Fusion Reactors vol. 2, 1996, Plenum Press, 559.

[9] T. Nishitani, T. Shikama, R. Reichle, E. R. Hodgson, E. Ishitsuka, S. Kasai, and S Yamamoto. In-situ irradiation test of mica substrate bolometer at the JMTR reactor for the ITER diagnostics. Fusion Eng. Des., 63-64:437-441, 2002.

[10] H. Langer, A. Steinbicker, H. Meister, and C. Zauner. Integrated thermal FE analyses and testing of prototype components for the ITER bolometer diagnostic. Fusion Eng. Des., 96-97:821-825, 2015.

[11] G. Nadasi, C. Gliss, H. Meister, A. Pataki, and F. Penzel. Conceptual studies for the management of thermal properties of ITER bolometer cameras. In $31^{\text {st }}$ Symposium on Fusion Technology (SOFT-31), Prague, Czech Republic, 2016. 